\title{
METALS AND SEMIMETALS WITH STRONGLY CORRELATED ELECTRONS
}

\author{
P. Fulde \\ Max-Planck-Institut für Physik komplexer Systeme \\ Bayreuther Str. 40, 01187 Dresden, Germany
}

\begin{abstract}
A characteristic feature of strongly correlated electrons is the appearance of low energy scales. They may result in heavy-quasiparticle excitations at low temperatures. Our understanding of these excitations has been considerably modified over the last few years. While it was originally thought that the Kondo effect is the sole source of heavy-fermion behavior, two additional mechanisms have been identified meanwhile. One is based on the Zeeman effect and occurs in $\mathrm{Nd}_{2-x} \mathrm{Ce}_{x} \mathrm{CuO}_{4}$. The other is operative in the semimetal $\mathrm{Yb}_{4} \mathrm{As}_{3}$ and is based on nearly half filled one-dimensional Hubbard chains. In all three cases the low energy excitations result from spin degrees of freedom.
\end{abstract}

PACS numbers: 71.27.+a, 75.20.Hr, 75.30.Mb, 71.10.Fd

\section{Introduction}

When we speak of metals with strongly correlated electrons we usually think of systems where the mutual Coulomb repulsions of electrons suppress considerably charge fluctuations on different atomic sites. The reductions can be so strong that a system may become insulating, even though from the point of view of band filling we would expect a metallic behavior. An often cited example is $\mathrm{La}_{2} \mathrm{CuO}_{4}$. With ionic charges $\mathrm{La}^{3+}$ and $\mathrm{O}^{2-}$ the $\mathrm{Cu}$ atoms are in a $\mathrm{Cu}^{2+}$ or $3 d^{9}$ configuration. Therefore we deal with a system which has one hole per formula unit and moreover, given the perovskite structure, per unit cell [1]. Therefore the system would be a metal were it not for the strong electron correlations which prevent it from being conducting.

A strong, though incomplete suppression of charge fluctuations has profound effects on the excitation spectrum of a system. Thereby we must distinguish between high- and low-energy excitations. High-energy excitations involve predominantly charge degrees of freedom. Moving an electron from one atom to another implies charge changes (or charge fluctuations) on the atoms involved. In the presence of large intraatomic Coulomb repulsions this implies large excitation energies. In the framework of a simple Hubbard Hamiltonian these transitions take place between the lower and the upper Hubbard band. 
Low-energy excitations on the other hand, often involve predominantly spin degrees of freedom. This is the case, e.g., in the heavy-fermion systems which are characterized by a very large density of states at small excitation energies $[2,3]$. Until recently the opinion was prevailing that all known heavy-fermion systems are the Kondo lattices. In a Kondo lattice a system of magnetic ions is coupled antiferromagnetically to nearly uncorrelated conduction electrons forming local singlets with them. Singlet-triplet excitations require small energies and provide for the high density of states in this case. However, in recent years it has turned out that heavy-fermion behavior can be of quite different origin. At least two other mechanisms, both involving spin degrees of freedom have been identified as generators of heavy quasiparticles. One is operative in $\mathrm{Nd}_{2-x} \mathrm{Ce}_{x} \mathrm{CuO}_{4}$ with $x$ around 0.2 [4] and is based on the Zeeman effect [5]. In distinction to a Kondo lattice system the conduction electrons are strongly correlated here, and this changes the underlying physics. A third mechanism is found in the semimetal $\mathrm{Yb}_{4} \mathrm{As}_{3}$ [6-10]. Here one is dealing with a system of chains with a band filling which is close to one half. The spin-like excitations of this system behave like neutral heavy fermions. It is likely that in the future other mechanisms will be identified which also result in heavy-fermion excitations.

\section{Heavy quasiparticle excitations}

As pointed out in the introduction there are presently three different mechanisms known, which can lead to heavy quasiparticles. We want to discuss them in the following. In particular, we want to point out the different physical phenomena associated with them.

\subsection{Kondo route to heavy fermions}

When magnetic ions like $\mathrm{Ce}$ or $\mathrm{Yb}$ are placed as impurities into a metal, the $4 f$ electrons hybridize weakly with the conduction electrons and form singlets. The Anderson impurity Hamiltonian

$$
\begin{aligned}
H= & \sum_{k m} \epsilon(k) c_{k m}^{\dagger} c_{k m}+\epsilon_{f} \sum_{m} n_{m}^{f}+\frac{U}{2} \sum_{m \neq m^{\prime}} n_{m}^{f} n_{m^{\prime}}^{f} \\
& +\sum_{k m} V(k)\left(f_{m}^{\dagger} c_{k m}+c_{k m}^{\dagger} f_{m}\right)+\widetilde{H}_{0}
\end{aligned}
$$

is usually used to describe this situation. The notation is the standard one and $\widetilde{H}_{0}$ contains all those degrees of freedom of the conduction electrons which do not couple to the impurity. The orbital index $m$ refers to the lowest $j$ multiplet. A variational ansatz for the ground state of that Hamiltonian is due to Varma and Yafet [11]. It is of the form

$$
\left|\Psi_{0}\right\rangle=A\left(1+\frac{1}{\sqrt{\nu_{f}}} \sum_{k m} \alpha(k) f_{m}^{\dagger} c_{k m}\right)\left|\Phi_{0}\right\rangle,
$$

in close analogy to one suggested by Yoshida [12] when the Kondo- instead of the Anderson Hamiltonian is considered. Here $A$ and $\alpha(k)$ are variational parameters, 
$\nu_{f}$ is the orbital degeneracy and $\left|\Phi_{0}\right\rangle$ represents the filled Fermi sea of conduction electrons. The ground state $\left|\Psi_{0}\right\rangle$ is a singlet and the energy gain due to the singlet formation is given by $k_{\mathrm{B}} T_{\mathrm{K}}$, where $T_{\mathrm{K}}$ is the Kondo temperature. The singlet-triplet excitation energy is often of the order of a few meV only. When a lattice of $\mathrm{Ce}$ or $\mathrm{Yb}$ ions is considered, like in $\mathrm{CeAl}_{3}, \mathrm{CeCu}_{2} \mathrm{Si}_{2}, \mathrm{YbCu}_{2} \mathrm{Si}_{2}$ or $\mathrm{YbAl}_{3}$, Hamiltonian (1) is replaced by the Anderson-lattice Hamiltonian. At low enough temperatures, i.e., $T<T_{\text {coh }}$ the different singlet-triplet excitations lock together and form coherent quasiparticle states. The surprising experimental observation is that there exists a nearly one to one correspondence between these excitations and those of a system of nearly free electrons with strongly renormalized mass. The implication is that we can treat the system like a Fermi liquid as described by Landau [13]. As a consequence we can calculate the Fermi surface of a heavy-fermion system like $\mathrm{CeRu}_{2} \mathrm{Si}_{2}$ or $\mathrm{CeCu}_{2} \mathrm{Si}_{2}$ by applying the renormalized band theory [14]. Thereby the effective potential acting on a quasiparticle is described in terms of energy dependent phase shifts $\eta_{l}^{A}(\epsilon)$. Here $A$ denotes the different atoms $A$ in the unit cell and $l$ is the angular momentum quantum number. The main idea is to use for these phase shifts the ones computed with a local density approximation (LDA) to density function theory except for the $l=3$ phase shifts of the rare earth (or actinide) ions. The latter are assumed to contain the strong electron correlations of the $4 f(5 f)$ electrons which the LDA is unable to describe. Within the spirit of Landau-Fermi liquid theory we expand therefore the phase shift $\eta_{l=3}^{\mathrm{Ce}(\mathrm{Yb})}(\epsilon)$ of the $\mathrm{Ce}$ or $\mathrm{Yb}$ ion around the Fermi energy $\epsilon_{\mathrm{F}}$ (more precisely, the phase shift corresponding to the crystal-field ground state $\tau$ of the lowest $j$ multiplet of the partially filled $4 f$ shell is expanded). We write, e.g.,

$$
\eta_{\tau}^{\mathrm{Ce}}(\epsilon)=\eta_{\tau}^{\mathrm{Ce}}\left(\epsilon_{\mathrm{F}}\right)+\frac{1}{k_{\mathrm{B}} T^{*}}\left(\epsilon-\epsilon_{\mathrm{F}}\right)
$$

and fix $\eta_{T}^{\mathrm{Ce}}\left(\epsilon_{\mathrm{F}}\right)$ by requiring a given number $n_{f}$ of $4 f$ electrons. Note that $n_{f}=1$ implies $\eta_{T}^{\mathrm{Ce}}\left(\epsilon_{\mathrm{F}}\right)=\pi / 2$. The only parameter entering the theory is $T^{*}$, which can be adjusted to the experimental specific heat coefficient $\gamma$. The temperature $T^{*}$ is here the analog of the single-ion Kondo temperature. Calculations of this form have predicted surprisingly well the large effective mass anisotropies in $\mathrm{CeRu}_{2} \mathrm{Si}_{2}$. For more details we refer to excellent reviews of Zwicknagl [15] and Norman and Koelling [16].

When the temperature increases, i.e., $T>T_{\text {coh }}$ the excitations lose their coherent properties and the problem reduces to that of independent impurities. For $T_{\text {coh }}<T<T^{*}$ the specific heat contains large contributions from the incoherent part of the $f$ electron excitations. In that regime the noncrossing approximation (NCA) may be used to describe the strongly correlated $f$ electrons and to explain a number of experiments [17].

Finally, when $T \geq T^{*}$ the Ce or $\mathrm{Yb}$ ions can be considered having a local moment which weakly couples to the conduction electron spins. Lowest-order perturbation theory suffices to describe that interaction. The range of application of the different theories is summarized in Fig. 1. A beautiful manifestation of this scenario is the experimentally observed difference in the Fermi surfaces of $\mathrm{CeRu}_{2} \mathrm{Si}_{2}$ and $\mathrm{CeRu}_{2} \mathrm{Ge}_{2}$. The main effect of replacing $\mathrm{Si}$ by $\mathrm{Ge}$ is an increase in the distance between $\mathrm{Ce}$ and its nearest neighbors. This is accompanied by a decrease in the 


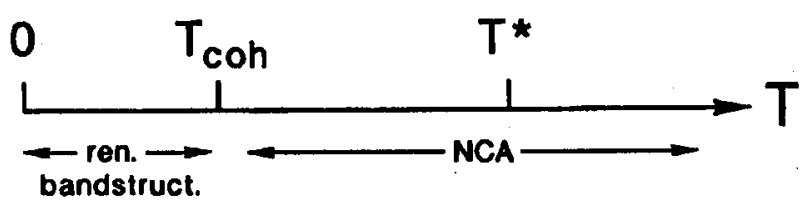

Fig. 1. Range of application of different methods for treating Kondo lattices.

hybridization of the $4 f$ electrons with the orbitals of the neighboring ions. While in $\mathrm{CeRu}_{2} \mathrm{Si}_{2}$ the characteristic temperature $T^{*} \approx 15 \mathrm{~K}$, it is practically zero in $\mathrm{CeRu}_{2} \mathrm{Ge}_{2}$. When de Haas-van Alphen experiments are performed at a temperature $T \approx 1 \mathrm{~K}$ this implies $T \ll T^{*}$ in $\mathrm{CeRu}_{2} \mathrm{Si}_{2}$ while for $\mathrm{CeRu}_{2} \mathrm{Ge}_{2}$ we have $T \gg T^{*}$. Therefore, in the former case the $4 f$ electron of Ce contributes to the volume enclosed by the Fermi surface, but not in the latter case. Indeed, measurements show that the two Fermi surfaces show a number of similar features but differ in volume by one electron.

\subsection{Zeeman route to heavy fermions}

The electron doped system $\mathrm{Nd}_{2-x} \mathrm{Ce}_{x} \mathrm{CuO}_{4}$ shows heavy-fermion behavior for concentrations in the range $0.1 \lesssim x \leqslant 0.2$ (see Fig. 2). For $x=0.2$ a linear specific heat coefficient $\gamma=4 \mathrm{~J} \mathrm{~mol}^{-1} \mathrm{~K}^{-2}$ is observed below $1 \mathrm{~K}$ [4]. The magnetic susceptibility $\chi_{\mathrm{s}}$ is approximately temperature independent in that regime and a Wilson ratio $R=\pi^{2} k_{\mathrm{B}}^{2} \chi_{\mathrm{s}} /\left(3 \mu_{\mathrm{eff}}^{2} \gamma\right)$ of order $R \approx 1.8$ is found. In distinction to the Kondo-lattice system $\mathrm{CeCu}_{2} \mathrm{Si}_{2}$ the linear specific heat at low temperatures in a $x=0.15$ sample remains virtually unchanged by the presence of superconductivity. Evidently, the heavy quasiparticles remain unaffected by the formation of Cooper pairs. There is strong experimental evidence that the low-energy excitations come from the magnetic degrees of freedom of the $\mathrm{Nd}$ ions. The $\mathrm{Nd}-\mathrm{Cu}$ exchange type interaction $H=\alpha \sum_{i, \delta} s_{i} S_{i+\delta}$ exceeds the one between the $\mathrm{Nd}$ ions and leads to the observed Schottky peak at $T \approx 2 \mathrm{~K}$ for the undoped $\mathrm{Nd}_{2} \mathrm{CuO}_{4}$ sample. Here $s_{i}$ is the spin of a $\mathrm{Cu}^{2+}$ ion at site $i$ and $S_{i+\delta}$ with $\delta=1,2$ denotes the spins of the two next-nearest $\mathrm{Nd}$ sites (the interactions between nearest $\mathrm{Cu}$ and $\mathrm{Nd}$ sites cancel each other due to the antiferromagnetic order of the $\mathrm{Cu}$ spins). From the position of the Schottky peak we can determine $\alpha$ and when we compute the Kondo temperature from it, we find that it is practically zero. In Fig. $2 a$ one notices that a Schottky like peak remains, even when the system shows no long-range order, i.e., for $x \gtrsim 0.1$. Apparently the $\mathrm{Cu}$ spins fluctuate sufficiently slowly in that case, so that the Nd spins can follow adiabatically that motion. Therefore they feel a molecular field acting on them and a spin flip costs Zeeman energy.

Electron doping changes some of the $\mathrm{Cu}^{2+}$ into $\mathrm{Cu}^{1+}$ ions which have a complete $3 d$ shell. The latter do not interact with the Nd spins. The $3 d^{10}$ configurations move in the $\mathrm{Cu}-\mathrm{O}$ planes and therefore the molecular field a $\mathrm{Nd}$ spin experiences is repeatedly turned on and off. This causes the large number of excitations on the low-energy side of the Schottky peak which give rise to the observed linear specific heat [5]. As is noticed from Fig. 2, a Schottky type peak is also found when the long-range order in the $\mathrm{Cu}-\mathrm{O}$ planes is destroyed by doping. This implies 


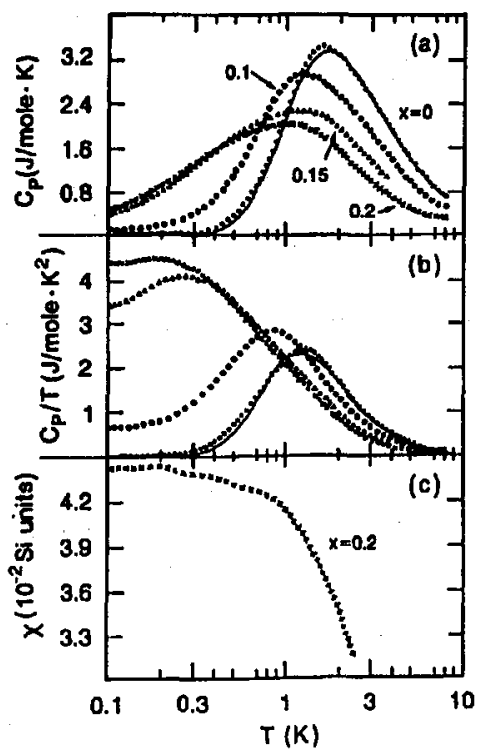

Fig. 2. (a) Specific heat $C_{p}(T)$ measurements for $\mathrm{Nd}_{2 \rightarrow x} \mathrm{Ce}_{x} \mathrm{CuO}_{4}$ for different electron-doping concentrations $x$. The solid line corresponds to a Schottky anomaly. (b) $C_{p}(T)$ as function of $T$. (c) Spin susceptibility $\chi(T)$ for $x=0.2$, i.e., in the overdoped regime (from [4], with authors' permission).

that appreciable short-range antiferromagnetic (AF) order is still present so that the Nd spins can follow (almost) adiabatically changes in the short-range order. This interpretation has been substantiated by inelastic neutron-scattering experiments [18] and by $\mu$ SR experiments [19]. In the former case, low-energy excitations corresponding to spin flips of the $\mathrm{Nd}$ ions are found also in $\mathrm{Nd}_{1.8} \mathrm{Ce}_{0.2} \mathrm{CuO}_{4}$, implying a molecular field seen by the $\mathrm{Nd}$ ions. In the $\mu \mathrm{SR}$ experiments a saturation of the relaxation time of the spin fluctuations is observed below $0.5 \mathrm{~K}$. It is of order $10^{-9} \mathrm{~s}$, even at $70 \mathrm{mK}$ and serves as direct proof that the Nd system is not a spin glass at those temperatures.

Two different model descriptions have been advanced to describe the observed heavy fermion behavior. One is using a spin-symmetry breaking, staggered field acting on the $\mathrm{Nd}$ spins in order to simulate the strong correlations of the conduction electrons and the resulting exchange field [5]. The corresponding Hamiltonian is

$$
\begin{aligned}
H= & -t \sum_{\langle i j\rangle \sigma}\left(a_{i \sigma}^{\dagger} a_{j \sigma}+\text { h.c. }\right)+h \sum_{i \sigma} \sigma \mathrm{e}^{\mathrm{i} Q \boldsymbol{R} \boldsymbol{R}_{i}} a_{i \sigma}^{\dagger} a_{i \sigma}+V \sum_{i \sigma}\left(a_{i \sigma}^{\dagger} f_{i \sigma}+\text { h.c. }\right) \\
& +\widetilde{\epsilon}_{f} \sum_{i \sigma} f_{i \sigma}^{\dagger} f_{i \sigma} .
\end{aligned}
$$

Here $a_{i \sigma}^{\dagger}, f_{i \sigma}^{\dagger}$ denote creation operators for conduction electrons and $4 f$ electrons, respectively. Furthermore, $\boldsymbol{Q}=(\pi, \pi)$ is a reciprocal lattice vector and $\boldsymbol{R}_{i}$ denotes the positions of the $\mathrm{Cu}$ ions. The $\mathrm{Nd}-\mathrm{Cu}$ is described by a hybridization 
term since it is easier to extract heavy quasiparticles from a Anderson-type than from a Kondo-type Hamiltonian. Iamiltonian (4) is easily diagonalized and yields two $d$-like $(\mathrm{Cu})$ and two $f$-like $(\mathrm{Nd})$ bands of excitations. Heavy quasiparticle excitations result here from a partially filled, narrow $f$ band. By adding an attractive interaction of the conduction electrons to the IIamiltonian we can show that the low-energy excitations and hence the linear specific heat are unaffected by superconductivity.

The second model description starts from a IIamiltonian

$$
H_{\text {int }}=J^{\prime} s \cdot S_{f}, \quad J^{\prime}>0,
$$

where $s$ is the spin of a Cu site and $\boldsymbol{S}_{\boldsymbol{f}}$ is the spin of an adjacent Nd ion [20]. Both spins are of magnitude $S$. The unit vector $\Omega=s / S$ is treated as a classical variable modulated by a stochastic interaction with the environment. It is $\langle\Omega(t)\rangle=0$ due to the absence of long-range order, while the correlation function is of the form

$$
\langle\boldsymbol{\Omega}(0) \boldsymbol{\Omega}(t)\rangle=\mathrm{e}^{-2 D_{\boldsymbol{r}} t} .
$$

Estimates of $D_{r}$ can be obtained from the nonlinear $\sigma$ model [21].

The stochastic motion of the spin $\boldsymbol{S}_{\boldsymbol{f}}$ obeys the equation

$$
\frac{\mathrm{d}}{\mathrm{d} t} n(t)=\omega_{0} \Omega(t) \times n(t),
$$

where $n(t)=S_{f}(t) / S$ and $\omega_{0}=J^{\prime} S$. The spectral function

$$
I(\omega)=\frac{1}{2 \pi} \int_{-\infty}^{+\infty} \mathrm{d} t \mathrm{e}^{\mathrm{i} \omega t}\langle n(0) n(\mathrm{t})\rangle
$$

can be determined under the assumption that the stochastic process is a Gaussian-Markoffian one, implying that the distribution function obeys a Fokker-Planck equation. As a result $I(\omega)$ is of the form

$$
I(\omega)=\frac{1}{3 \pi} \frac{4 D_{r}}{\omega^{2}+\left(4 D_{r}\right)^{2}}+\left(\text { side peaks at } \omega_{0}\right) \text {. }
$$

For undoped $\mathrm{Nd}_{2} \mathrm{CuO}_{4}$ the rate $D_{r}$ vanishes at low temperatures $T$ because of Néel order. When the system is doped, we expect that $D_{r}$ remains finite when $T \rightarrow 0$. A finite value of $I(\omega)$ around $\omega=0$ gives rise to a contribution of the spin $S_{f}$ to the specific heat of the form $C=\gamma T$. This follows from

$$
C(T)=\frac{\mathrm{d}}{\mathrm{d} T}\left\langle H_{\text {int }}\right\rangle=\frac{S(S+1)}{T^{2}} \int_{0}^{\infty} \mathrm{d} \omega \omega^{2} I(\omega) \frac{1}{\cosh ^{2}(\omega / 2 T)} .
$$

The side peaks in Eq. (9) give rise to a Schottky peak in $C(T)$. When the $f$ spin susceptibility is calculated, it is found that $\chi(T) \sim \ln \left(D_{r} / T\right)$ at low $T$. This shortcoming is most likely due to the neglect of quantum fluctuations which reduce $\chi(T)$ at low $T$. But it is interesting to note that for $\mathrm{Nd}_{1.8} \mathrm{Ce}_{0.2} \mathrm{CuO}_{4}$ and $T=0.4 \mathrm{~K}$ the present approach leads to a Sommerfeld-Wilson ratio $R=$ $\pi^{2} \chi /\left(3 g^{2} \mu_{\mathrm{B}}^{2} \gamma\right) \approx 1.4$. Here $g^{2}=1.27$ is the squared average of the Nd Landé factor. The net result is therefore that a stochastic, classical approach describes the specific heat data of $\mathrm{Nd}_{1.8} \mathrm{Ce}_{0.2} \mathrm{CuO}_{4}$ very well but not the spin susceptibility, which requires the inclusion of quantum fluctuations. 


\section{3. $Y b_{4} A s_{3}-$ an unusual semimetal}

The intermetallic compound $\mathrm{Yb}_{4} A s_{3}$ crystallizes in the anti-Th $\mathrm{P}_{4}$ structure. In that structure the $\mathrm{Yb}$ ions occupy four sets of parallel chains which point along the diagonals of a cube. The structure should be compared with the one of $A 15$ compounds for which $\mathrm{V}_{3} \mathrm{Si}$ is an example. Here the $\mathrm{V}$ ions occupy three sets of parallel chains which point along the main axes of a cube.

With a valency of 3 - the three As ions attract 9 electrons and therefore the four $\mathrm{Yb}$ ions must have valencies $3 \times \mathrm{Yb}^{2+}+1 \times \mathrm{Yb}^{3+}$. The ion $\mathrm{Yb}^{2+}$ has a filled $4 f$ shell while that of $\mathrm{Yb}^{3+}$ has one hole. As this hole is shared between four $\mathrm{Yb}$ ions, the system is metallic at high temperatures. However, at $T_{\mathrm{s}} \approx 300 \mathrm{~K}$ it undergoes a trigonal distortion in a weak first-order structural phase transition. The resistivity changes discontinuously at that temperature and increases with decreasing $T$ until it reaches a maximum of approximately $10 \mathrm{~m} \Omega \mathrm{cm}$ at $T \approx 130 \mathrm{~K}$. At very low temperatures it obeys a law $\rho(T)=\rho_{0}+A T^{2}$, indicating Fermi liquid behavior. Measurements of the Hall constant reveal a dramatic increase below $T_{s}$, indicating that the system becomes a semimetal at low temperatures. In fact, at low $T$ there remains only one charge carrier per $10^{3} \mathrm{Yb}$ ions. Despite the low number of carriers, the low temperature specific heat has a linear specific heat coefficient of order $\gamma \approx 200 \mathrm{~mJ} /\left(\right.$ mole $\left.\mathrm{K}^{2}\right)$. The spin susceptibility $\chi_{\mathrm{s}}$ is Pauli like and large with a Sommerfeld-Wilson ratio of order unity. This clearly indicates heavy-fermion behavior. This is further substantiated by the observation that the ratio $A / \gamma^{\nu}$ $(\nu \approx 2)$ is similar to that of other heavy-fermion systems [6,22]. Due to the low carrier concentration the heavy quasiparticles must certainly result from spin degrees of freedom. The Kondo effect can be excluded as origin of the observed heavy-fermion behavior. The low energy scale corresponding to the observed $\gamma$ value is of order $T \approx 40 \mathrm{~K}$. Inelastic neutron scattering shows clearly a crystal field level of $\mathrm{Yb}^{3+}$ at an excitation energy of approximately $40 \mathrm{~K}$ which would not be the case if Kondo singlets were formed. The neutron results are in agreement with Mößbauer data on ${ }^{170} \mathrm{Yb}$ which give evidence that below $50 \mathrm{~K}$ perfect charge ordering takes place.

In order to explain the above experimental findings the following theory has been advanced [9]. At $T_{\mathrm{s}}$ a collective band Jahn-Teller (CBJT) phase transition takes place. It corresponds to a volume conserving distortion by which one family of chains (e.g., in 〈111〉 direction) is shrinking, while the other three families of chains are elongating. The transition is triggered by a strong deformation-potential coupling, which is commonly found in mixed valence systems. It splits the fourfold degenerate quasi-1D density of states into a nondegenerate and a triply degenerate one. The nondegenerate density of states corresponds to the family of shortened chains. Because of their smaller ionic radius the $\mathrm{Yb}^{3+}$ collect in the short chains while the $\mathrm{Yb}^{2+}$ go into the long chains. Thus charge ordering takes place with decreasing temperatures. At $T=0$ we expect almost perfect charge ordering in which case the system would be a one-dimensional Mott-Hubbard insulator. The fact that it is a semimetal and not an insulator is likely to be related to the nonvanishing hopping matrix elements between $4 f$ orbitals in the short and long chains. This may result in weak self-doping, since some of the holes in $4 f$ shells 
move from the short into the long chains. The CBJT can be described by an effective Hamiltonian of the form

$$
H=-t \sum_{\mu} \sum_{\langle i j\rangle \sigma}\left(f_{i \mu \sigma}^{\dagger} f_{j \mu \sigma}+\text { h.c. }\right)+\epsilon_{\Gamma} \sum_{\mu i \sigma} \Delta_{\mu} f_{i \mu \sigma}^{\dagger} f_{i \mu \sigma}+4 N_{L} c_{0} \epsilon_{\Gamma}^{2} .
$$

Here $f_{i \mu \sigma}^{\dagger}$ creates an $f$ hole with spin $\sigma$ at site $i$ in chain $\mu$. The notation $\langle i j\rangle$ refers to nearest neighbors within a chain of $N_{\mathrm{L}}$ sites and interchain hopping is neglected here. The trigonal-strain order parameter is denoted by $\epsilon_{\Gamma}$, with a corresponding bulk elastic constant $4 c_{0}$. The deformation potential $\Delta_{\mu}$ is

$$
\Delta_{\mu}=\Delta\left\{\begin{aligned}
1, & \mu=1 \\
-\frac{1}{3}, & \mu=2,3,4 .
\end{aligned}\right.
$$

Note that Eq. (11) does not yet contain the strong on-site repulsions of $4 f$ electron, which are responsible for Mott-Hubbard insulators. Close to $T_{\mathrm{s}}$ those interactions are not important though. With $4 t=0.2 \mathrm{eV}$ (from LDA calculations), $c_{0}=10^{11} \Omega \mathrm{erg} / \mathrm{cm}^{3}$, where $\Omega$ is the volume of a unit cell, and $\Delta=5 \mathrm{eV}$, one obtains $T_{\mathrm{s}} \approx 250 \mathrm{~K}$.

At low $T$, where the $f$ electron correlations are important, a $t$ - $J$ Hamiltonian is used. By applying a slave-boson mean field approximation one can formally derive quasiparticle excitations with the observed heavy masses. However, a calculation of this kind does not bring out clearly the physical origin of the heavy quasiparticles. Because of the low carrier concentration they must come from the spin excitations in the short chains. Although no long-range magnetic order has been detected down to $1 \mathrm{~K}$ [7], the short range antiferromagnetic correlations lead to spin-wave like excitations which we can describe by linear spin-wave theory. This picture has been experimentally confirmed by Kohgi et al. who measured spin-wave spectra by inelastic neutron scattering and found that they are equal to those of a 1D spin chain [23]. It is well known that in one dimension, spin-wave excitations lead to a linear specific heat. There exists a one to one correspondence between them and excitations of a weakly interacting electron system with a strongly renormalized mass. Therefore we may speak of heavy fermions, but since the system is almost insulating it is more appropriate to speak of a heavy neutral Fermi liquid. This physical interpretation allows for an explanation of another experimental observation. In an external magnetic field of $4 \mathrm{~T}$ the $\gamma$ coefficient in the specific heat changes considerably below $2 \mathrm{~K}$ [24]. This is unexpected, since we would expect the changes to be of order $\left(\mu_{\mathrm{B}} H / k_{\mathrm{B}} T^{*}\right)^{2}$ where $T^{*} \approx 40 \mathrm{~K}$ is the characteristic low energy scale. These findings can be explained by taking into account a weak coupling between the short chains [10]. Within spin-wave theory ratio of $10^{-4}$ between interchain and intrachain coupling explains the experiments due to the opening of an anisotropy gap.

\section{Conclusions}

During the last few years new experimental findings have shown that heavy quasiparticles can be of different physical origin. Three physical effects have been identified until now leading to heavy-fermion behavior. The classical one is based on the formation of Kondo singlets and applies to systems like $\mathrm{CeAl}_{3}, \mathrm{CeCu}_{2} \mathrm{Si}_{2}$ or 
$\mathrm{CeRu}_{2} \mathrm{Si}_{2}$. A second mechanism is operative in $\mathrm{Nd}_{2-x} \mathrm{Ce}_{x} \mathrm{CuO}_{4}(0.1<x \leq 0.2)$ and is based on the Zeeman effect. A characteristic feature of the system are the strong correlations of the conduction electrons in the $\mathrm{Cu}-\mathrm{O}$ planes. Finally, in the semimetal $\mathrm{Yb}_{4} \mathrm{As}_{3}$ we are dealing with Hubbard chains close to half filling. Despite the low carrier concentration of typically one carrier per $10^{3} \mathrm{Yb}$ ions the linear specific heat coefficient $\gamma$ is several hundred times as large as, e.g., in Na metal. In all three cases the low-energy excitations giving rise to the large $\gamma$ values involve predominantly spin degrees of freedom. For Kondo lattices they are singlet-triplet excitations, in $\mathrm{Nd}_{2-x} \mathrm{Ce}_{x} \mathrm{CuO}_{4}$ they involve spin flips of the $\mathrm{Nd}$ ions while.in $\mathrm{Yb}_{4} \mathrm{As}_{3}$ they consist of spin flips in the short chains consisting of $\mathrm{Yb}^{3+}$ ions. The question is legitimate whether or not the correspondence to excitations of almost free electrons is exact or approximate only in the three cases. While no clear cut answer can be given, the experimental evidence is in favor of a reasonable close mutual correspondence. There is no reason that the mechanisms described here are the only ones leading to heavy quasiparticles. We speculate that in $U$ compounds showing heavy-fermion behavior there are still other physical effects at work.

\section{Acknowledgment}

I would like to thank J. Igarashi, B. Schmidt, P. Thalmeier, V. Zevin and G. Zwicknagl for fruitful collaboration.

\section{References}

[1] N.M. Plakida, High-Temperature Superconductivity, Springer, Berlin 1995.

[2] G.R. Steward, Prog. Low Temp. Phys. 56, 755 (1984).

[3] P. Fulde, J. Keller, G. Zwicknagl, in: Solid State Physics, Vol. 41, Eds. H. Ehrenreich, D. Turnbull, Academic Press, San Diego 1988, p. 1.

[1] T. Brugger, T. Schreiner, G. Roth, P. Adelmann, G. Czjzek, Phys. Rev. Lett. 71, $2481(1993)$.

[5] P. Fulde, V. Zevin, G. Zwicknagl, Z. Phys. B 52, 133 (1993).

[6] A. Ochiai, T. Suzuki, T. Kasuya, J. Phys. Soc. Jpn. 59, 4129 (1990).

[7] P. Bonville, A. Ochiai, T. Suzuki, E. Vincent, J. Phys. I 4, 595 (1994).

[8] P.H. Reinders, U. Ahlheim, K. Fraas, F. Steglich, T. Suzuki, Physica B 186-188, 434 (1993).

[9] P. Fulde, B. Schmidt, P. Thalmeier, Europhys. Lett. 31, 323 (1995).

[10] B. Schmidt, P. Thalmeier, P. Fulde, Europhys. Lett., in press.

[11] C. Varma, Y. Yafet, Phys. Rev. B 13, 2950 (1976).

[12] K. Yoshida, Phys. Rev. 147, 223 (1966).

[13] L.D. Landau, Zh. Eksp. Teor. Fiz. 30, 1058 (1956) [Sov. Phys.-JETP 3, 920 (1957)]; Zh. Eksp. Teor. Fiz. 32, 59 (1957) [Sov. Phys.-JETP 5, 101 (1957)].

[14] H. Razafimandimby, P. Fulde, J. Keller, Z. Phys. B 54, 111 (1984).

[15] G. Zwicknagl, Adv. Phys. 41, 203 (1992).

[16] M.R. Norman, D. Koelling, in: Handbook on the Physics and Chemistry of Rare Earths, Vol. 17, Eds. K.A. Gschneidner, L. Eyring, G.H. Lander, G.R. Choppin, Elsevier, Amsterdam 1993, p. 1. 
[17] For a review see N.E. Bickers, Rev. Mod. Phys. 59, 845 (1987).

[18] M. Loewenhaupt, A. Metz, N.M. Pyka, D.M. Paul, J. Martin, V.H.M. Duijn, J.J.M. Trause, H. Mutka, W. Schmidt, Ann. Phys. (Germany) 5, 197 (1996).

[19] J. Litters, private communication.

[20] J. Igarashi, K. Murayama, P. Fulde, Phys. Rev. 52, 15966 (1995).

[21] S. Chakravarty, B.I. Halperin, D.R. Nelson, Phys. Rev. B 39, 2344 (1989);

S. Chakravarty, R. Orbach, Phys. Rev. Lett. 64, 224 (1990).

[22] T. Kasuya, J. Phys. Soc. Jpn. 63, 2481 (1994).

[23] M. Kohgi, private communication.

[24] R. Helfrich, F. Steglich, A. Ochiai, to be published. 